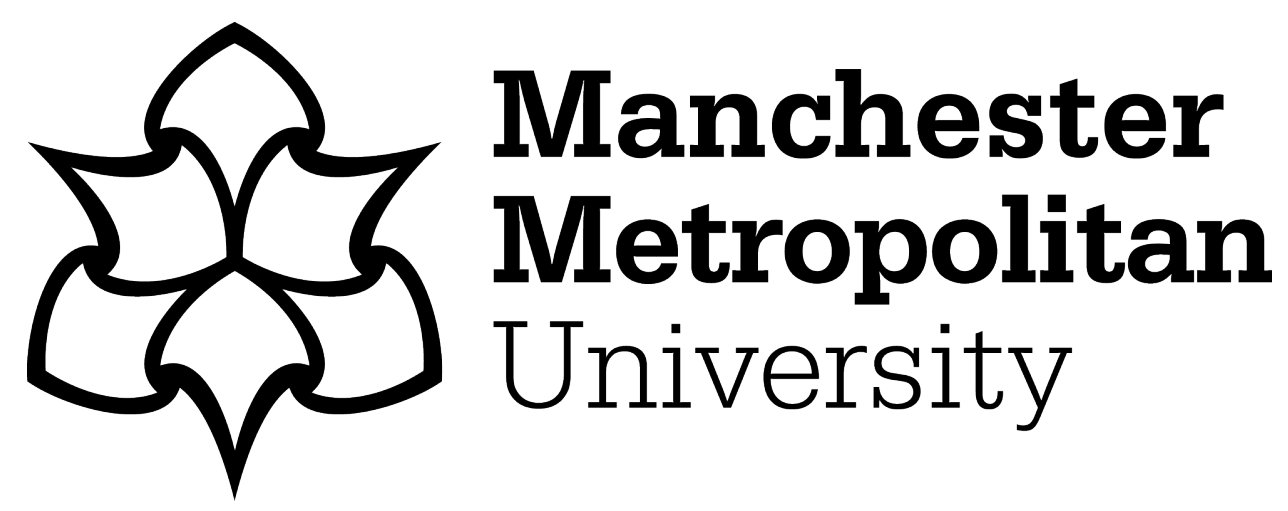

Salmon, Neil (2017) Outdoor Passive Millimeter Wave Imaging: Phenomenology and Scene Simulation. IEEE Transactions on Antennas and Propagation, 66 (2). pp. 897-908. ISSN 0018-926X

Downloaded from: https://e-space.mmu.ac.uk/619728/

Version: Published Version

Publisher: IEEE

DOI: https://doi.org/10.1109/TAP.2017.2781742

Usage rights: Creative Commons: Attribution 3.0

Please cite the published version 


\title{
Outdoor Passive Millimeter-Wave Imaging: Phenomenology and Scene Simulation
}

\author{
Neil A. Salmon ${ }^{\mathbb{1}}$
}

\begin{abstract}
A model for scene simulation is presented, which describes quantitatively the recognized phenomenology of passive millimeter-wave (PMW) imaging in land, sea, and air scenarios. The model describes objects in a scene as facets giving them electromagnetic properties from their electrical permittivities. This enables polarimetric effects in scenes to be reproduced. Illumination from the sky and ground is calculated using radiation transport, as are the emissive and absorbing effects of the atmosphere between the objects and the imager. The model includes the effects of image sampling, diffraction effects of finite imager aperture sizes, and system noise. Phenomena in experimentally acquired images in the $94 \mathrm{GHz}$ atmospheric transmission window are quantified from three scenarios. These are a helicopter landing site as viewed by the pilot, a coastal region showing boats, land and buildings viewed from sea level, and an airfield viewed from directly above. These are compared quantitatively to the simulated scenes from these scenarios, finding agreement between the radiation temperatures to within a few degrees kelvin. These discrepancies are accounted for by the uncertainties in the permittivities of materials in the scene. This paper constitutes the partial validation of a scene simulation capability for PMMW imaging in outdoor scenarios.
\end{abstract}

Index Terms - Imaging, millimeter-wave technology, radio propagation, radiometry, simulation.

\section{INTRODUCTION}

A MOTIVATION for operating in the millimeter waveband is that atmospheric obscurants (of fog, cloud, falling rain, falling snow, smoke, and brownout) have a high transmission $(>50 \%)$, whereas in the classical imaging bands of the midinfrared and visible band, transmission is only a fraction of a percent. Other bands such as the terahertz band (above a few hundred $\mathrm{GHz}$ ) become almost unusable at sea level at ranges beyond a few tens of meters, due to the high $(>10 \mathrm{~dB} / \mathrm{m})$ atmospheric absorption, even in clear air.

Over the past 35 years, there has been a huge diversity of passive millimeter-wave (PMMW) imaging systems, which have probed the $10-500 \mathrm{GHz}$ frequency band to establish the phenomenology and sensing capabilities. The large data set of published imagery from this can now be used to validate scene simulation models in order that new measurement scenarios can be effectively investigated, initially without the requirements for costly trials. A number of other models for outdoor

Manuscript received May 23, 2017; revised August 26, 2017; accepted October 18, 2017. Date of publication December 14, 2017; date of current version February 1, 2018.

The author is with the Faculty of Science and Engineering, Manchester Metropolitan University, Manchester M15 6BH, U.K. (e-mail: n.salmon@mmu.ac.uk).

Color versions of one or more of the figures in this paper are available online at http://ieeexplore.ieee.org.

Digital Object Identifier 10.1109/TAP.2017.2781742 scene simulation have been developed [1]-[3] and initial work on this model began with [4].

Material properties and illuminations govern the appearance of objects in the scene. Metals reflect $\sim 100 \%$ of incident radiation and transmit almost nothing, as the electromagnetic skin depth is typically hundreds of nanometers. Dielectrics partially reflect, transmit, and emit. When they contain water they become more reflective, and less transmissive and emissive. Water is both partially emissive and reflective, the relative portions being a strong function of radiation frequency. Trees, foliage, and grass have emissivities in the region of $95 \%$ and reflect and scatter only $5 \%$.

In Section II, the physical effects of objects and their illumination on an outdoor environment are discussed. This describes the ray-tracing scene simulation model that uses the known material properties of objects and calculates their illumination from the environment. Section III describes the instrument effects of finite imager size, spatial sampling in the image, and the effects of system noise. Section IV presents experimentally acquired imagery in the $94 \mathrm{GHz}$ band for a range of land, sea, and air remote sensing scenarios. For these scenarios, the $94 \mathrm{GHz}$ atmospheric transmission window is the optimal frequency band for sensors having apertures of up to a meter and delivering spatial resolution of a few meters. This arises due to the competing factors of higher atmospheric transmissions at lower frequencies and better spatial resolutions at higher frequencies [5]. Although there is an atmospheric transmission window centered on $140 \mathrm{GHz}$, outdoor PMMW imagery has significantly reduced contrast due to the higher atmospheric absorption, while at $35 \mathrm{GHz}$ the spatial resolution is poorer [6]. For this reason there is a relatively large published experimental data set of images at $\sim 94 \mathrm{GHz}$, which have been exploited to validate scene simulation. Phenomenology is discussed and simulated scenes representative of the experimental data are provided, making quantitative comparisons and assessing systematic uncertainties. Section V presents an overall conclusion of this paper.

\section{Physical EFFEcts of OBJects And Illumination}

To model objects in a scene, there must be a quantitative description of the illumination of objects, how the objects interact with this illumination and how the atmosphere between the objects and the imager affect the appearance of the objects. The physics of these interactions are now described.

\section{A. Radiometric Emission Levels From Materials}

PMMW imaging is the science of imaging the radiometric (or thermal emission) from objects. The level of thermal 
emission from an object is given by the product of its emissivity and the Planck blackbody function corresponding to its temperature. Emissivity varies from zero (for objects which are completely reflective) to unity (for objects which have no reflection). The level of spectral radiance given from the Planck blackbody function, in units of Watts per area of radiating surface at temperature $T$, per steradian of space, and per unit radiation bandwidth (into both polarizations) at a wavelength $\lambda$ is

$$
I(T, \lambda)=2 \frac{h f}{\lambda^{2}} \frac{1}{\exp (h f / k T)-1}
$$

where $f$ is the radiation frequency and $c=f \lambda$, where $c$ is the speed of light, $h$ is the Planck constant, and $k$ is Boltzmann's constant [7]. In the spectral regime where the thermal energy of the source $(k T)$ is significantly greater than the photon energy $(h f),(1)$ simplifies to that of the RayleighJeans formula, namely

$$
I(T, \lambda)=2 \frac{k T}{\lambda^{2}} .
$$

This equation holds for the majority of terrestrial sources well below the discrimination frequency defined by $h f=k T$, which corresponds to a wavelength of $10 \mu \mathrm{m}$ for ambient temperature sources. This regime, therefore, includes the terahertz band, the submillimeter band, the millimeter waveband and radio frequencies below these. To achieve the best possible spatial resolution, the zero-order diffraction mode from the imager is selected, whereby from first principles the product of the source radiating area and the solid angle becomes $\lambda^{2}$. The highest through-put efficiency is achieved by transition selecting a single polarization. Under these conditions, the Planck blackbody function power in watts entering the receiver becomes

$$
P\left(T, B_{\mathrm{RF}}\right)=k T B_{\mathrm{RF}}
$$

where $B_{\mathrm{RF}}$ is the radio frequency bandwidth, normally defined by the receiver front-end components. This power is independent of the radiation frequency, because it represents the power received in a single mode of the imager.

Calibration of the power measured by imaging radiometers is normally performed by measuring the emission from large area (thereby ensuring the filling of the antenna pattern) blackbody radiators, at two known temperatures, as described in [9]. One of these is usually at ambient temperature and the other at liquid nitrogen temperature. When convenient the emission from the zenith sky can be used in place of the liquid nitrogen source, as the sky has a relatively low emission level, which is stable and known. Because of the simplicity of the relationship of (3), it is customary to characterize emission levels in PMMW imaging in terms of the "temperature" of this emission, which is referred to as the radiation temperature of the source, having units of kelvin.

\section{B. Objects and Backgrounds Modeled as Facets}

A convenient source of 3-D models for scene simulations arises due to the computer graphics industry. Models of almost any 3-D object are available. They can be placed in a scene and the facets of the object assigned the physical properties of the material.

The ray-tracing model of this paper identifies facets in a scene, determines their illumination and uses the orientations of facets to determine their polarimetric reflectance, transmittance, and emission. Reflectance can be calculated by using Fresnel's equations or Lambert's scattering law, or determined by bi-directional reflection coefficient measurements. The absorption is calculated using Beer's law [8], where the facets are ascribed a thickness and permittivity. In the ray-tracing scheme, a "ray" begins its journey at the imager location, moves in a direction given by the direction vector $\mathbf{V}$, passes through the atmosphere (suffering absorption and emission through radiation transport). Upon encountering a facet, it is reflected in a direction $\mathbf{R}$ defined by

$$
\mathbf{R}=\mathbf{V}-2 \hat{\mathbf{n}}(\hat{\mathbf{n}} \cdot \mathbf{V})
$$

where $\hat{\mathbf{n}}$ is the unit direction vector of the facet normal. After reflection the ray continues its journey searching for further facets that will be intersected. If no further facets are encountered the background emission from the particular ray direction is added. Ray-tracing is performed on the two orthogonal linear polarizations from the imager and polarimetric effects of facet reflection are determined by decomposing these polarizations into perpendicular and parallel components at the facet. The "ray" moves in exactly the opposite direction to the millimeter-wave radiation.

In the scenarios described here the thicknesses of all objects are sufficiently large that no radiation is transmitted. Only a single ray reflection from a facet is considered to keep computation to a minimum. Modeling of multiple reflections is necessary for some scenarios where there is emission from cavities [10].

\section{Reflectance, Transmittance, and Emissivity of Facets}

In the scenarios considered in this paper materials are nontransmissive; their thicknesses and absorptions are sufficiently high that no millimeter-wave radiation passes through these objects. Under these conditions, the radiation temperature of the facet $T_{F}$ is given by

$$
T_{F}=T_{\mathrm{BR}} \Gamma+T_{0} E
$$

where $T_{\mathrm{BR}}$ is the background illuminating radiation temperature impinging on the facet from the direction vector $\mathbf{R}$, and $\Gamma$, $E$, and $T_{0}$ are reflectance, emissivity, and ambient temperature of the facet, respectively. As the facet transmittance is assumed to be zero, the reflectance and emissivity are related through the conservation of energy by

$$
1=\Gamma+E \text {. }
$$

This simply means that radiation emanating from a facet originates either from a reflection of incident radiation or by the (Planck) radiative emission process within the facet. No radiation incident on the reverse side of the facet is transmitted through the facet. This is a reasonable and valid assumption for many of the materials encountered in the outdoor, land, sea and air scenarios modeled in this paper. 
TABLE I

Relative Permittivities of Materials Modeled in the Scene Simulations Derived From a VAriety of Sources, Assumed FOR SimUlations AT OVER THE 90-94 GHz BAND

\begin{tabular}{|c|c|}
\hline Material & Relative permittivity $\varepsilon_{\mathrm{r}}$ or reflectance $\Gamma$ \\
\hline Metal & $\Gamma=100 \%$ (specular when surface smooth) \\
\hline Water (at $15{ }^{\circ} \mathrm{C}$ ) [22] & $\varepsilon_{\mathrm{r}} \sim(6.57,11.17)$ \\
\hline Concrete [24] & $\varepsilon_{\mathrm{r}} \sim(6.19,0.34)$ \\
\hline Fiberglass [25] & $\varepsilon_{\mathrm{r}} \sim(4.49,0.161)$ \\
\hline Sand [26] & $\varepsilon_{\mathrm{r}} \sim(4.5,0.5)$ \\
\hline Sandstone [27] & $\varepsilon_{\mathrm{r}} \sim(4.05,0.05)$ \\
\hline Asphalt [28] & $\varepsilon_{\mathrm{r}} \sim(3.18,0.10)$ \\
\hline Brick [25] & $\varepsilon_{\mathrm{r}} \sim(2.55,0.43)$ \\
\hline Nitrile rubber [29] & $\varepsilon_{\mathrm{r}} \sim(2.65,0.032)$ \\
\hline Wood [30] & $\varepsilon_{\mathrm{r}} \sim(1.82,0.12)$ \\
\hline $\begin{array}{c}\text { Grass or deciduous tree } \\
\text { covered land [31] }\end{array}$ & $\Gamma \sim 5 \% \quad($ Lambertian) \\
\hline
\end{tabular}

Experimental polarimetric imagery indicates measured radiation temperatures in horizontal and vertical polarizations are different [11], [13]. These differences are described by the Fresnel equations [14] of (7) and (8), which give different reflection coefficients $r_{\perp}$ and $r_{\|}$for radiation with electric field vectors perpendicular $(\perp)$ and parallel $(\|)$ to the plane of incidence of the ray at the facet surface

$$
\begin{aligned}
r_{\perp} & =\frac{\cos \theta_{I}-n \cos \theta_{T}}{\cos \theta_{I}+n \cos \theta_{T}} \\
r_{\|} & =\frac{\cos \theta_{T}-n \cos \theta_{I}}{\cos \theta_{T}+n \cos \theta_{I}} .
\end{aligned}
$$

In these equations, $n$ represent the complex refractive index of the material surface (having real $n^{\prime}$ and imaginary $n^{\prime \prime}$ components) and $\theta_{I}$ and $\theta_{T}$ are the angles of incidence and transmission, respectively, measured from the facet normal. They are related through Snell's law $\sin \theta_{I} / \sin \theta_{T}=n^{\prime}$. The reflectance is given by the complex square of the amplitude reflection coefficient, namely, $\Gamma=r r^{*}$ where ${ }^{*}$ denotes the complex conjugate.

Since the beginning of the 1990s, a number of PMMW imaging systems have demonstrated that many man-made materials such as concrete, asphalt, building tiles, and bricks are specular reflectors at $94 \mathrm{GHz}$ [15], [19]. This might be expected as the surface roughness of these materials is generally less than the wavelength of the millimeter-wave radiation. The phenomenon arising from this is that radiation from these surfaces becomes partially polarized, which is why these surfaces often appear with objects reflected in them.

Materials are characterized by the relative complex permittivity $\varepsilon_{r}$ (having real $\varepsilon_{r}^{\prime}$ and imaginary $\varepsilon_{r}^{\prime \prime}$ components), which has the complex square relation $\varepsilon_{r}=n^{2}$ to the refractive index. For the small data set of materials included in these simulations, the relative permittivities from a variety of difference sources are presented in Table I.
Changes in complex permittivity of materials with temperature and radiation frequency are related to how bound and free charges in the medium respond to the oscillating field of an electromagnetic wave [29]. Bound charges respond by undergoing relaxation or resonance orientation processes, where the characteristic frequencies of these physical processes depend on intermolecular forces. Likewise, free charges respond by an oscillatory linear motion.

In the case of pure water, the movements of bound and free charges introduce relatively large changes in the complex permittivity. This behavior has been modeled empirically [20], [23]. At $94 \mathrm{GHz}$, these indicate that pure water at a temperature of $0{ }^{\circ} \mathrm{C}\left(25{ }^{\circ} \mathrm{C}\right)$ has a complex permittivity of 5.65 and 7.86 (7.97 and 14.71). However, at $35 \mathrm{GHz}$, the permittivity is larger, becoming 10.04 and 19.98 (22.50 and 31.35) at $0{ }^{\circ} \mathrm{C}\left(25^{\circ} \mathrm{C}\right)$. In the case of water, the change with temperature arises due to the fall in the viscosity with increased temperature. This enables the bound charges of the water molecule to orientate more quickly with the electric field of wave. Permittivity has higher values at lower frequencies, as the bound charges have more time to orientate themselves to the field. The characteristic relaxation frequency of pure water is approximately $16.7 \mathrm{GHz}$ at $20{ }^{\circ} \mathrm{C}$. This frequency increases with temperature and if saline is introduced into the water.

In the case of sea water, this has values of permittivity a few percent lower than those of pure water at $94 \mathrm{GHz}$. This effect is influenced by the motion of the free ions resulting from dissolved solids, mainly sodium chloride [23].

Any material capable of holding a variable amount of water will also likewise show a variation in permittivity with frequency and temperature. Such materials are permeable and include for example, many types of rock, most bricks, and wood. Other materials generally show less variability in permittivity with temperature and frequency.

An increase in permittivity will lead to a higher reflectivity, so objects reflecting "cold" sky radiation will appear with lower radiation temperatures. Owing to such effects, it is always advisable to use measured complex permittivity values appropriate for the temperature of the object and the radiation frequency band of the simulation.

The radiation bandwidth of sensor systems operating in the $94 \mathrm{GHz}$ transmission window is unlikely to exceed $\sim 30 \%$. There are two reasons for this. First, systems are designed to fit into the transmission window [5]. Expansion beyond this into the absorption bands would be detrimental to performance. Second, antennas with bandwidths larger than $30 \%$ tend to have higher losses and lower aperture efficiencies. This results in higher effective noise and poorer spatial sampling of the radiation field in the focal plane array or the aperture, resulting in lower performance.

When surfaces have a roughness or unevenness approaching the wavelength they generally exhibit a fraction of the polarization effects that are predicted by the Fresnel equations. The roughness effectively scrambles the polarization. This behavior can be described by introducing into the model a polarization scrambling fraction $p$ (ranging from 0 to 1 ). The radiation temperatures of the perpendicular and parallel polarizations 
after scrambling $T_{\perp, S}$ and $T_{\|, S}$ are given by (9) and (10) where $T_{\perp}$ and $T_{\|}$are the radiation temperatures before scrambling. When there is no scrambling $p=0$ then $T_{\perp, S}=T_{\perp}$ and $T_{\|, S}=T_{\|}$and when there is complete scrambling $p=1$ and $T_{\perp, S}=T_{\|, S}=\left(T \perp+T_{\|}\right) / 2$. Given the knowledge of the surface properties of objects, the degree of polarization can be adjusted to bring the simulated images in line with the measured images within reasonable limits

$$
\begin{aligned}
T_{\perp, S} & =\frac{1}{2}\left[T_{\perp}(2-p)+T_{\|} p\right] \\
T_{\|, S} & =\frac{1}{2}\left[T_{\perp} p+T_{\|}(2-p)\right] .
\end{aligned}
$$

For a surface having a roughness, which is far greater than the wavelength, it will scatter a plane incident wave in all directions, which is the exact opposite of specular reflection. Under these conditions it may be assumed (or confirmed by measurement) that incident radiation at a particular polarization is scattered by equal amounts into the perpendicular and parallel polarizations. A convenient quantitative description of this behavior is Lambertian scattering, in which the radiant intensity of a surface is proportional to the cosine of the viewing angle, with respect to the facet normal. It has been confirmed by many measurements that grass and deciduous trees behave as a Lambertian scatterer in the millimeter waveband with a reflectance of the order of $5 \%$, as illustrated in Table I. This is also supported by airborne nadir measurements [32], which indicate the emissivity of vegetation and soil at 24, 50, and $89 \mathrm{GHz}$ to be in the region of $95 \%$.

When the surface roughness lies in the intermediate regime, the characteristics of the reflection are between that of a specular reflector and a Lambertian scatterer. A plane wave incident on a surface is usually reflected at a distribution of angles about the specular angle of reflection $\theta_{R}\left(=\theta_{I}\right)$. Under these conditions it is advisable to experimentally characterize this reflectivity distribution by what is referred to as a bidirectional polarimetric reflectivity measurement. Here the polarimetric reflectance at a particular angle of reflection is measured for each angle of incidence and polarization.

\section{Limitation of Nontransmissive/Nonscattering Facets}

Limitations of this particular model are that the objects are assumed to consist of facets that are nontransmissive. This is equivalent to saying the reflectance and emissivity sum to unity, as indicated in (6). This is valid provided the scale length over which intensity falls to a fraction $1 / e$ is smaller than the material thickness, which is generally the case for materials of interest in this paper and given in Table I.

There may well be interests in exploiting other attributes of the millimeter waveband, such as recognizing objects that may be (intentionally) concealed by a visibly opaque material, which maybe semitransparent to millimeter waves. The semitransparency of materials has been modeled [33] by introducing semitransparent facets, in which the thickness of the material is an additional specified parameter. This then enables the transmittance of these facets to be calculated. This has been used in the scene simulation of screening people for threats concealed under clothing. In a remote sensing scenario, it may be used to recognize decoys or threats concealed under camouflage nets. In the ray-tracing scenarios, rays propagate to these semitransparent facets and are partially reflected and transmitted, before being reflected by underlying facets and transmitted back through the semitransparent facets, in the direction of the imager. This adds considerable complexity to the model and for brevity is not further discussed.

\section{E. Atmospheric Absorption and Radiation Transport}

For a PMMW imager viewing an object the effect of atmospheric attenuation is to attenuate the signal from the object and to generate additional emission from the atmosphere. These two contributions are quantified, respectively, by the following equation [34]. An object having a radiation temperature $T_{\text {OBJECT }}$ will appear with a radiation temperature at the imager of

$$
T_{\text {IMAGER }}=T_{\text {OBJECT }} \exp \left(-\tau_{0}\right)+\int_{0}^{\tau_{0}} T_{\text {ATM }} \exp (-\tau) d \tau
$$

where $T_{\mathrm{ATM}}$ is the temperature of the atmosphere. The quantity $d \tau$ is the increment in the optical depth, this being the product of the atmospheric absorption coefficient and the path length increment. The optical depth $\tau$ is the line integral of this quantity. The full optical depth $\tau_{0}$ is [34] the line integral of the absorption coefficient from the imager to the object, namely

$$
\tau_{0}=-\int_{\text {IMAGER }}^{\text {OBJECT }} \kappa_{\mathrm{ATM}} d s .
$$

The absorption and emission arising from the radiation transport is calculated by splitting up the atmosphere of the earth into layers having different temperatures, pressures, and water vapor densities at each altitude. Validated models of atmospheric temperature, pressure, and water vapor content are available for different regions of the globe [35]. Validated models of the absorption coefficients for these conditions are available from the International Telecommunications Union (ITU) recommendations for clear atmosphere [36], foggy and cloudy atmospheres [37], and atmospheres with rainfall [38]. Models are emerging for absorption in brownout conditions [39], [42].

\section{F. Object Illumination and Background}

For outdoor scenarios objects will be illuminated by natural radiation from the environment, this is reflected from objects in the direction of the imager. This illumination is dominated by the low level of radiation temperatures from the sky over $\sim 2 \pi$ steradians, which has a minimum at the zenith and increasingly higher levels when moving toward the horizon. The radiation temperatures of the earth are generally higher than that of the sky and are defined by the material properties of the earth's surface, its physical temperature, and the illuminating radiation temperature of the sky.

If there are no objects in the immediate vicinity of a region and the ground is uniform, the illumination in that region is symmetrical about an axis from the zenith to the nadir. This can be used as an assumption in first-order calculations of 
the illumination of objects and in the creation of the image background. By tracing rays at angles between the zenith and the horizon the illumination from the sky can be estimated. This is done using (11) to calculate the atmospheric emission out to an altitude of $30 \mathrm{~km}$, beyond which any residual atmospheric emission is a small fraction of a kelvin. A residual value of $2.7 \mathrm{~K}$ is added for the cosmic background radiation. The emission from the atmosphere is unpolarized.

The radiation temperature of the ground for zenith angles from the horizon to the nadir can be calculated by assuming the surface of the earth to be at ambient temperature and have known specular or Lambertian reflection properties. As illumination from the zenith to the horizon is known, this becomes the illumination of the surface of the earth. This is then used to calculate the illumination from the horizon to the nadir, assuming reflection properties of the earth and taking into consideration radiation transport absorption and emission in the atmosphere. As illumination from the earth comes from a surface, it may be partially polarized through the Fresnel equations.

The illumination that is down welling from the sky and up welling from the surface of the earth forms the full illumination profile, which is used to calculate the illumination of objects and the image background. This illumination is a function of the millimeter-wave frequency. Moving into an absorption band, or generally up in frequency, increases the atmospheric absorption and this raises the illumination radiation temperature toward the ambient temperature. Calculations are made in this frequency for the $94 \mathrm{GHz}$ band and examples of the illumination profiles for the three scenarios are displayed as a function of zenith angle in the following sections.

Although indoor scene simulation is outside the remit of this publication, it is instructive to consider how the illumination might be in such scenarios. Basically, for an indoor scenario, if there is no "cold" sky radiation entering through windows or open doors, the illuminating radiation temperature will be that of the inside building temperature, this level being fixed by the thermostatically controlled air conditioning, at around $22{ }^{\circ} \mathrm{C}$. Therefore, people inside those buildings will appear as higher radiation temperature objects. The radiation temperature contrast between them and the inside of the building will be $\left(1-R_{H}\right)\left(T_{H}-T_{0}\right)$, where $R_{H}$ and $T_{H}$ are the reflectivity and temperature of the human body, respectively, and $T_{0}$ is the ambient temperature in the building. This temperature contrast is much smaller (typically 6 to $12 \mathrm{~K}$ ) than those of the outdoor scenarios, but has been exploited for security screening of people and modeled in [10].

\section{G. Rendering}

Rendering of objects in the scene is performed by combining the effects of the object illumination, the polarimetric reflection from faceted objects and radiation transport. The scene is completed by adding a background, which is generated from the illumination profile at the imager location.

\section{INSTRUMENT EFFECTS}

A rendered scene is that which is effectively presented to the imager. From thereon, instrumental effects such as sampling, the diffraction associated with a finite aperture size, and the system noise now need to be added.

\section{A. Choice of Radiation Frequency}

The simulations in this paper have been performed around $94 \mathrm{GHz}$, because at this frequency there is a cross section of reliable experimental imagery from various systems in different scenarios. The $94 \mathrm{GHz}$ band is an attractive frequency, because for meter-sized objects viewed from imagers having apertures of this size, there is a good compromise between angular resolution and atmospheric attenuation. For this reason, simulations have to be performed with a simulated point spread function for this aperture size at this frequency. Higher frequencies promise better spatial resolution, but practically this is never realized due to the poor contrast arising from higher atmospheric absorption. At lower frequencies, higher contrast may be achieved for larger objects, but features of many objects of interest will not be seen as they are below the diffraction limited spatial resolution.

Focal plane array imagers with apertures larger than $\sim 1 \mathrm{~m}$ become impracticable due to their bulkiness and the availability of space on the majority of platforms. Aperture synthesis imagers employing conformally distributed receivers may enable larger apertures, and hence better spatial resolutions [43], [45]. This may shift optimal performance to the lower radiation frequencies.

\section{B. Sampling the Image}

Sampling of the rendered image is made according to the type of imager. The angular sample size for a focal plane array imager is defined by the feed spacing at the focus [5] and for an aperture synthesis imager it is defined by the array geometry, as discussed in [45]. To achieve optimal performance, systems should sample at least at the Nyquist spatial frequency of the Airy disk pattern in the focal plane [8]. This corresponds to an angular sample size on the rendered image of half the diffraction limited angular resolution, $\lambda / 2 D$ where $D$ is the physical aperture size of the imager.

\section{Diffraction-Convolution With the Point Spread Function}

The diffractive effects of the finite aperture size and wavelength of radiation are included by convolving the sampled rendered image with the point spread function of the optical system. Either an estimated or measured point spread function can be used. For simulations in this paper, a point spread function for an optical system having a $1 \mathrm{~m}$ diameter aperture was used.

\section{Radiometric Sensitivity and System Noise}

To complete the simulation, noise generated by the electronics in the system is added to each pixel of the simulated image. The electronics of a PMMW imager comprise a cascade of low noise amplifiers, sometimes mixers, detectors, and analog-todigital-convertors, each making their own noise contribution. The low-noise amplifiers and mixers generate Johnson, shot, carrier recombination, and fluctuation noise. The amplifiers 
also make an effective noise contribution by their fluctuations in gain, the level of which under some circumstances can be minimized or even removed. In a well-designed system, the amplifier gains are raised until the front-end amplifier noise dominates. However, there usually remains some residual flicker or $1 / f$ noise generated by the detector. Since the key parameter being measured here is radiation temperature, noise contributions are quantified in terms of noise equivalent temperature deviation, in units of kelvin. As these noise contributions are random and independent, their sum total can be written [34] as a root-mean-square (rms) combination

$$
\Delta T_{T}^{2}=\Delta T_{I}^{2}+\left(T_{\mathrm{SYS}} \frac{\Delta G}{G}\right)^{2}+\Delta T_{1 / f}^{2}
$$

where the first term is the ideal noise of a radiometer, the second term is the contribution due to the fluctuations $\Delta G$ in the amplifier power gain $G$, and the final term is the flicker noise. In the above, $T_{\mathrm{SYS}}$ is the system noise temperature, which is the sum of the average radiation temperature in the scene and the receiver noise temperature. A study of this noise in a $94 \mathrm{GHz}$ monolithic microwave integrated circuit receiver focal plane array indicates the combined first and second contributions are about $60 \%$ larger than the final term contribution in (13). As the noise level in (13) is an rms value of an assumed Gaussian distribution, a random number generator is then used to add a small deviation in radiation temperature to each image pixel of the scene.

The ideal noise of a radiometer system $\Delta T_{I}$ is determined [47] by the radiometer equation

$$
\Delta T_{I}=\frac{T_{A}+T_{N}}{\sqrt{B_{\mathrm{RF}} t_{I}}}
$$

where $T_{A}$ is the antenna temperature (essentially the radiation temperature of the scene in front of the antenna), $T_{N}$ is the noise temperature of the radiometer receiver, $B_{\mathrm{RF}}$ is the radio frequency bandwidth, and $t_{I}$ is the integration time of the single channel receiver.

In a focal plane array imager, a number of receiver channels measuring the radiometric intensity $N_{R}$ are scanned over a number of pixels in the scene $N_{P}$ in an image acquisition frame time $t_{F}$. As the system noise is random and uncorrelated between channels, the ideal radiometric noise at the pixel level, from the radiometer equation (14) becomes

$$
\Delta T_{I}=\frac{T_{A}+T_{N}}{\sqrt{B_{\mathrm{RF}} t_{F}}}\left(\frac{N_{P}}{N_{R}}\right)^{1 / 2} .
$$

In an aperture synthesis imager having $N_{R}$ receiver channels measuring the complex electric field of the radiometric emission, the ideal radiometric noise is given [49] by

$$
\Delta T_{I}=\frac{T_{A}+T_{N}}{\sqrt{B_{\mathrm{RF}} t_{F}}} \frac{1}{F}
$$

where $F$ if the fractional filling of the aperture with the receiver antennas, given by

$$
F=\left(\frac{N_{R} A_{R}}{A_{S}}\right)
$$

where $A_{R}$ is the collection area of a single antenna in the array and $A_{S}$ is the synthesized area of the complete array. Given the number of pixels generated by the aperture synthesis imager is just the ratio of $A_{S}$ to $A_{R}$, the ideal radiometric noise at the pixel level of the aperture synthesis system becomes

$$
\Delta T_{I}=\frac{T_{A}+T_{N}}{\sqrt{B_{\mathrm{RF}} t_{F}}}\left(\frac{N_{P}}{N_{R}}\right) .
$$

Comparing (18) with (15), it is seen they differ by a factor $\sqrt{ }\left(N_{P} / N_{R}\right)$. This is because in the focal plane array imager, the intensity from a resolution beam-with is sampled, while in the aperture synthesis imager, the electric field from the complete field of view is sampled. In both cases, the receiver noise temperatures can be measured using standard radiometry methods [47].

Radiometric noise levels of the order of $1 \mathrm{~K}$ have been demonstrated for both focal plane array and aperture synthesis imagers. Falling component costs over the next few years will enable larger radiometric bandwidths in aperture synthesis imagers and greater numbers of channels to deliver system sensitivities of the order of $100 \mathrm{mK}$ at $25 \mathrm{~Hz}$ frame rates. For this reason this level of noise has been added to the simulated images in this paper.

\section{E. Presenting Easy-to-Interpret Images-Contrast Inversion}

The majority of PMMW images of outdoor scenes are displayed using the convention that on a gray scale, higher radiation temperatures are darker and lower radiation temperatures are lighter. This is referred to as the black is radiometrically "hot" convention. Using this convention the sky appears bright, as do upper surfaces reflecting "cold" sky radiation. The underside surfaces of objects in PMMW images have higher radiation temperatures, so appear dark. Using this convention means PMMW images show striking resemblances to images in the visible band, and thereby quick and easy to interpret, an important aspect for machine-human interface.

In cases where the majority of the scene has radiation temperatures approaching ambient, with only a small number, of very tiny objects with low radiation temperatures, the convention of black is radiometrically "cold" is used. For these scenarios, this convention is more favorable, as the majority of the simulated scene appears with a lighter shade. The radiometric temperatures in the simulations in this paper are shown by a contrast bar above the images.

Analysis of on-screen displayed images can be made by running the cursor over regions of interest to read out directly on the screen the radiation temperatures, precise to a small fraction of a kelvin. Alternatively the image can be written to a file for later analysis or approximate values may be estimated by comparing the shade in the image with the contrast bar above the image.

\section{Modeled Outdoor Scenes Compared With EXPERIMENTALLY ACQUIRED IMAGERY}

To illustrate the phenomenology of outdoor PMMW imaging, three scenarios have been modeled and the results compared with experimental imagery. The three scenarios are: 1) a helicopter landing scene as viewed by the pilot; 2) boats, land, and buildings in a coastal environment as viewed from the 


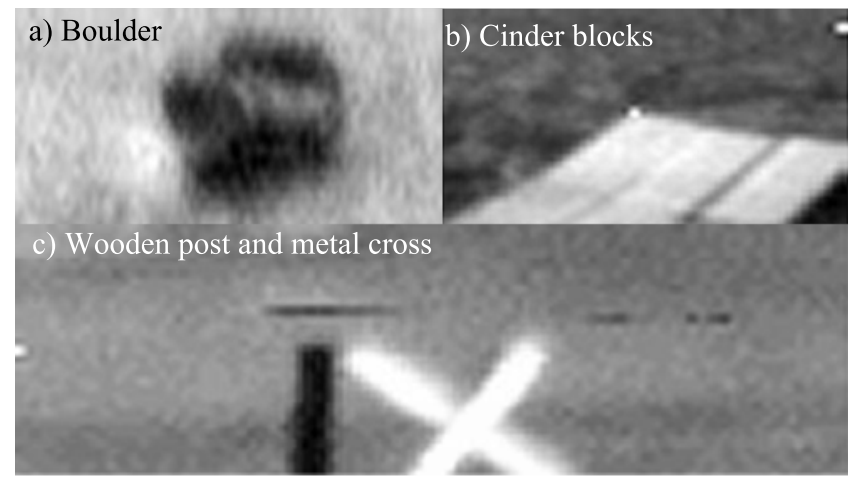

Fig. 1. Extracts of measured imagery in the horizontal polarization from [50] showing the (a) boulder, (b) cinder blocks, and (c) wooden post with the octahedral metal cross. Black denotes radiometrically 'hot.'

shore; and 3) an airfield as viewed at the nadir from directly above. To the end of this section, the systematic uncertainties associated with the simulations are discussed.

The levels of radiation temperature recorded by the groups acquiring the experimental imagery have been taken directly from their publications. The temperatures have been taken from their presented imagery contrast bars, graphs of temperature versus azimuth, and specific statements in the texts. Experimental data in the imagery are normally calibrated using ambient and liquid nitrogen temperature absorbers [9], as indicated in Section II. When performed by competent operators, calibration accuracy is normally precise to within a degree kelvin or so.

\section{A. Helicopter Landing Scenario}

The helicopter benefits from a PMMW imager as situational awareness is required in visually degraded environments, such as landing in fog or rotor down-thrust-induced dust storms (referred to as brownout), when in close proximity to obstacles [48]. Some excellent quantitative experimental measurements of such obstacles have been made [50] in clear weather that can be used in the partial validation of scene simulation. Obstacles measured in this paper were cinder blocks, a boulder, a vertically standing wooden post, and an octahedral metal cross, all located on a sand base with an asphalt road to the right-hand side. Extracts of images of these made at $94 \mathrm{GHz}$ in the horizontal polarization are presented in Fig. 1.

Simulation of these objects is performed assuming the PMMW imager has an aperture of $1 \mathrm{~m}$ in diameter, located at an altitude of $15 \mathrm{~m}$, looking down at a depression angle of $29^{\circ}$, with an azimuth/elevation field of view of $22^{\circ} / 12^{\circ}$. Experimental measurements were performed during a hot period when the daytime temperature was in the region $303.15-306.15 \mathrm{~K}$ $\left(30{ }^{\circ} \mathrm{C}-33{ }^{\circ} \mathrm{C}\right)$ and the physical temperature of the background sand in direct sunlight became as high as $323.15 \mathrm{~K}\left(50^{\circ} \mathrm{C}\right)$ and the sky radiation temperature, measured at a zenith angle of $60^{\circ}$, was $190 \mathrm{~K}$ [50]. These data were used to fix the sky illumination profile from the zenith to the horizon using a standard model of the atmosphere [35], which is shown in Fig. 2.

In the simulation modelled at $94 \mathrm{GHz}$ the background viewed at a zenith angle of $120^{\circ}$ (a depression angle of $30^{\circ}$ ) the

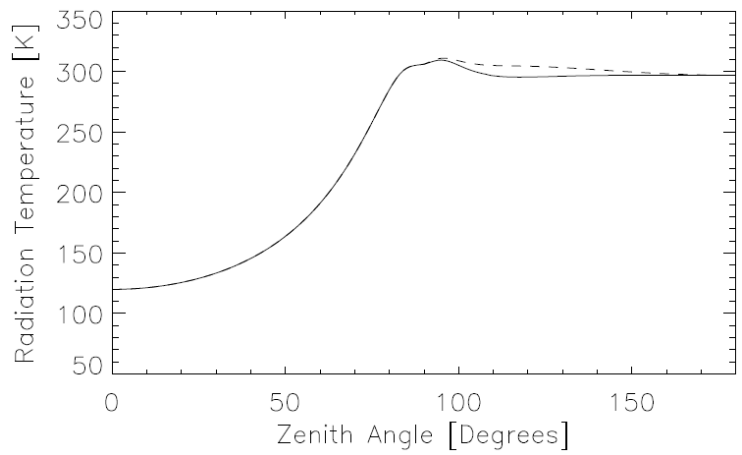

Fig. 2. Illumination (sky and ground) radiation temperature profile as a function of zenith angle from $0^{\circ}$ (zenith) to $90^{\circ}$ (horizon) and the ground illuminating radiation profile based on a sand background from the horizon to the $180^{\circ}$ (nadir) for horizontal (solid line) and vertical polarization (dashed line).

radiation temperature is 295.3 and $304.1 \mathrm{~K}$ for the horizontal and vertical polarizations, respectively, which are the same values as in the measurements. This was achieved assuming that the sand surface was a specular reflector with a complex relative permittivity of $(4.5,0.5)$ and that the surface introduced an $80 \%$ fraction of polarization scrambling. The permittivity determines level of emission and the fraction of scrambling determines the difference between the levels in the two polarizations.

This knowledge about the sand enables the remainder of the illumination profile from the horizon to the nadir to be determined and this is shown in Fig. 2. In the portion of the profile from zenith angles of $100^{\circ}$ to $150^{\circ}$, the temperature difference between the horizontal and vertical polarization rises to $\sim 10 \mathrm{~K}$, which is the same as that in the measurement [50].

The boulder was simulated as sandstone having a complex relative permittivity of $(4.05,0.05)$ with a $50 \%$ fraction of polarization scrambling and a physical temperature of $310 \mathrm{~K}$ $\left(37{ }^{\circ} \mathrm{C}\right)$. This produces radiation temperatures on the front of the boulder of 302.7 and $308.5 \mathrm{~K}$ in the horizontal and vertical polarizations, as shown in Fig. 3, compared to the measured values of 298 and $303 \mathrm{~K}$. These values also reproduce the experimental observation that there is little radiation temperature contrast between the top of the boulder and the surrounding sand.

A shadow cast by the boulder seen on its left-hand side, from the sun shining from the right-hand side, can be seen as a lighter shade in the measured imagery of Fig. 1. From the azimuthal traces of radiation temperatures in [50], the radiation temperature of the shadow is $\sim 3.5 \mathrm{~K}$ lower than that of the surrounding sand, in both polarizations. The simulation of the shadow was made by reducing the physical temperature of the sand by $4.5 \mathrm{~K}$ on the left-hand side of the boulder, which reduces the radiation temperature of this area of background sand by $\sim 3.5 \mathrm{~K}$. This lower radiation temperature can be seen as a lighter shade on the left-hand side of the boulder in the simulated imagery in Fig. 3.

The cinder blocks were simulated as having a permittivity of that of concrete 6.19 and 0.34 and having a physical temperature of $321 \mathrm{~K}$. This generated radiation temperatures of 283.5 and $304.4 \mathrm{~K}$ for the horizontal and vertical polarizations for the upper surface of the blocks, while the measure- 

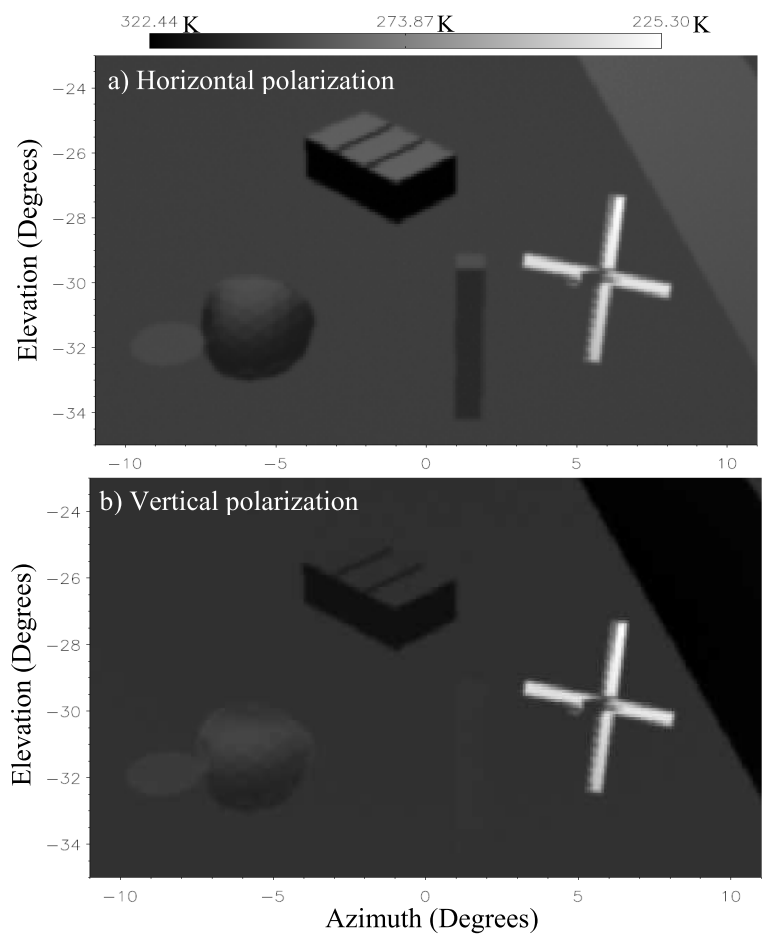

Fig. 3. Simulation of a helicopter landing site showing (from left to right) potential obstacles of a boulder, cinder blocks, a wooden post, an octahedral metal cross on a sand base, and a section of asphalt road in the (a) horizontal polarization and (b) vertical polarization. Black is radiometrically "hot," the contrast bar at the top indicates radiation temperatures in kelvin.

ments indicated values of 279 and $300 \mathrm{~K}$, respectively. In the simulation, the contrast between the background sand and the top of the blocks in the vertical polarizations is less than $0.1 \mathrm{~K}$, and not detectable in the simulated image of Fig. 3, or in the experimental data of [50]. The front of the cinder blocks appears with a radiation temperature of 317.5 and $316.3 \mathrm{~K}$ in the horizontal and vertical polarizations, respectively, seen in high contrast against the sand background, this also being noted in measurements of [50].

The wooden post was simulated with a permittivity of 1.82 and 0.12 , a value derived from [30] and a physical temperature of $303.15 \mathrm{~K}\left(30{ }^{\circ} \mathrm{C}\right)$. This generated simulated radiation temperatures for the horizontal and vertical polarizations of the vertical side of the post of 302.9 and $303.1 \mathrm{~K}$, whereas the measured radiation temperature was $300 \mathrm{~K}$. The contrast between the post and the background sand is $-0.8 \mathrm{~K}$, so it is barely visible in the simulated image, but unfortunately there is no measured image of the post in the vertical polarization in [50] for comparison.

The metal octahedral cross is the object in the scene with the lowest radiation temperature. As the cross comprises three orthogonal bars, its surfaces are reflecting slightly different parts of the sky, having slightly different illuminating radiation temperatures. The lowest radiation temperature on the cross is $225.5 \mathrm{~K}$ in both polarizations. This agrees well with the measured value of $225 \mathrm{~K}$ from [50].

\section{B. Coastal Maritime Environment}

The maritime environment is characterized generally by a high relative humidity, particularly in the evaporation duct, a layer approximately up to $20 \mathrm{~m}$ thick above the sea surface.

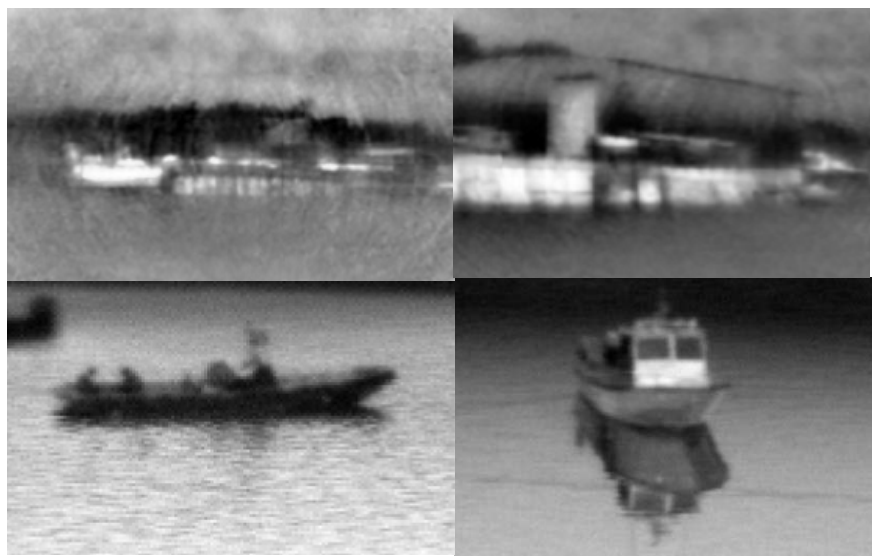

Fig. 4. Measured imagery in the horizontal polarization showing a metal boat with brick buildings in the background (top left) [54], metal pilings (top right) [54], a rubber boat (lower left) [53], and a fiber glass boat (lower right) [52]. Black denotes radiometrically 'hot.'

The littoral region, extending from the shore to the edge of the continental shelf, can suffer coastal (advection) fog as a result of warm humid air passing into cooler regions. The high humidity and fog gives rise to poor visibility and infrared imagers are frequently incapable of penetrating these obscurants. Transmission of millimeter-wave radiation under these conditions is relatively good [51] and consequently PMMW imaging provides situational awareness in these scenarios.

Some spectacular imagery at $94 \mathrm{GHz}$ of fiberglass and rubber boats from [52] and [53] and a metal boat from [54] are shown in Fig. 4. These demonstrate characteristic features for this scenario, namely, that metal boat hulls and harbor pilings have low radiation temperatures against higher radiation temperatures of background water. In addition, rubber boats have high radiation temperatures against the lower radiation temperature background water. A feature observed in the image of the metal boat is that the background land, which is covered primarily with brick buildings, has high radiation temperatures. The fiberglass boat has radiation temperatures intermediate between that of the metal and the rubber boat. In the calm water of this image a specular reflection of the boat can be seen in the water surface. The description of reflections in plane surfaces is not covered in this paper, but a model for this has been described in [10].

For the maritime environment the ITU mean annual global reference atmosphere profile [35] is used, which has a sea level air temperature of $288.15 \mathrm{~K}\left(15^{\circ} \mathrm{C}\right)$, pressure of $1013 \mathrm{mbar}$, and a water vapor density of $7.5 \mathrm{~g} / \mathrm{m}^{3}$. Through atmospheric radiation transport, these determine the illumination profile from the zenith to the horizon at $94 \mathrm{GHz}$, as shown in Fig. 5 . A completely plane sea surface (having no waves) is modeled as the background and used to calculate the remainder of the illumination radiation temperature profile from the horizon to the nadir.

For the purpose of simulating the costal environment, boats comprising four different materials together with a piece of land with a building on it have been selected for simulation. The results for this simulation are shown in Fig. 6. The materials from which the boats are constructed are fiberglass, wood, nitrile rubber and metal. The land is assumed to be 


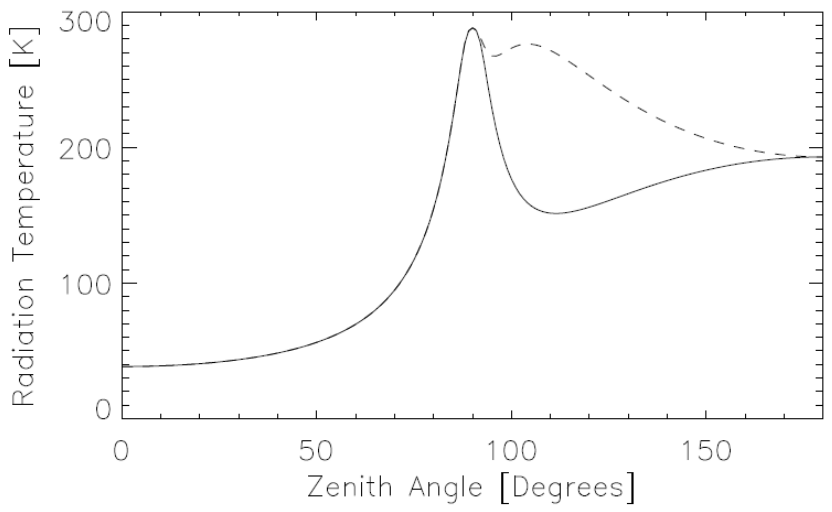

Fig. 5. Illumination (sky and ground) radiation temperature profile at sea level for the maritime scenario with the sea as the background as a function of zenith angle, for the horizontal (solid line) and vertical (dashed line) polarizations. For zenith angles less than $90^{\circ}$, the horizontal and vertical polarizations have the same radiation temperature.

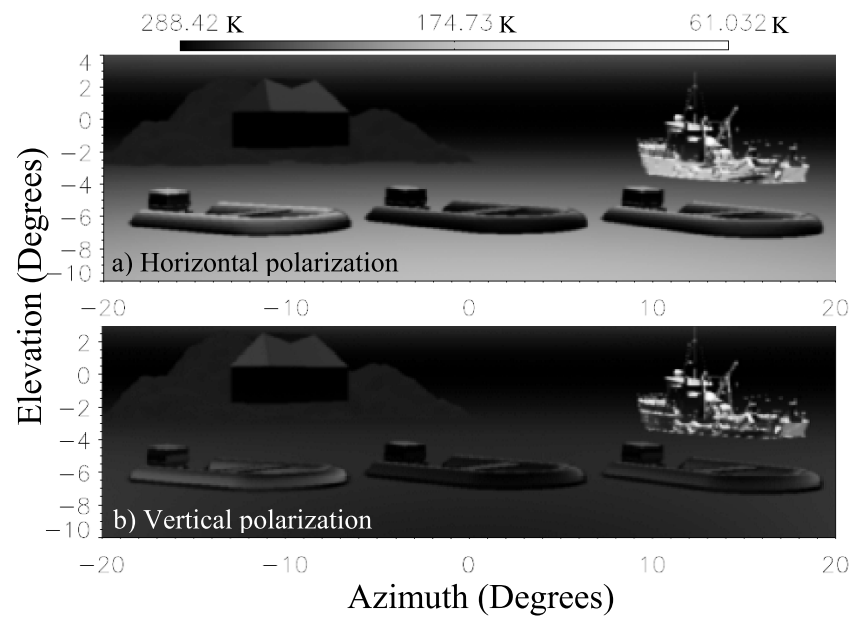

Fig. 6. Simulation of small boats (from left to right made from fiberglass, wood, and nitrile rubber), a large metal vessel, a land mass, and a building with a pitched roof in the maritime environment in the (a) horizontal polarization and (b) vertical polarization. The scale above represents the scene radiation temperature in kelvin; black denotes radiometrically 'hot'; the lower and side scales represent the azimuth and elevation in degrees.

covered in grass and the building is assumed to be made of clay bricks with a clay tiled, pitched roof.

A characteristic of all outdoor PMMW imagery, seen more clearly from the coast is a dark band of high radiation temperatures around the horizon. This can be seen in the illumination profile in Fig. 5 and as a dark band of radiation along the horizon of the simulated images of Fig. 6. The dark band exists because rays heading for the horizon have the longest optical path length through the earth's atmosphere. These then generate the highest radiation temperatures through radiation transport of (11).

A polarimetric characteristic is also present in the illumination welling up from the water surface and this can be observed in Fig. 5 for zenith angles between $90^{\circ}$ and $180^{\circ}$. Radiation temperature in the vertical polarization from the sea surface is characteristically higher than that of the horizontal polarization, as shown in the illumination profile of Fig. 5. As seen from Fig. 5, the maximum difference between the vertical and horizontal polarization radiation temperatures is $\sim 125 \mathrm{~K}$, occurring at a zenith angle of $110^{\circ}$. The maximum in radiation temperature of the vertical polarization occurs at a zenith angle of $103^{\circ}$. This corresponds to the Brewster incidence angle $\left[\arctan \left(n^{\prime}\right)\right]$ of $77^{\circ}$ at the sea surface for a relative permittivity of 6.57 and 11.17 for water. The effect of the higher radiation temperature in the vertical polarization can also be seen as a darker shade from the simulation of the water surface in Fig. 6. This Brewster angle effect is present in other scenes where there is specular reflection from materials, such as for example concrete and asphalt.

Of the small nonmetallic boats, that with the highest radiation temperature is made from wood (the center boat in Fig. 6). This is because, from the Fresnel equations, this has the lowest reflectivity. The small boat with the lowest radiation temperature is made from fiberglass (to the left-hand side of Fig. 6), as this has the highest reflectivity. The small nitrile rubber boat (to the right-hand side of Fig. 6) has radiation temperatures somewhere between these. Brewster angle effects means radiation temperatures of these boats are higher in the vertical polarization than in the horizontal polarization, as illustrated in the simulation of Fig. 6 .

The front facing surface of the nitrile rubber boat has a radiation temperature in the range from 267.3 to $283.2 \mathrm{~K}$, with the adjacent water having a radiation temperature of $201.4 \mathrm{~K}$, in the horizontal polarization. In the vertical polarization the boat radiation temperatures are in the range from 278.4 to $283.5 \mathrm{~K}$ with the adjacent water having a radiation temperature of $266.8 \mathrm{~K}$. The simulation indicates the rubber boat appears as having higher radiation temperatures than the surrounding water, which is what the experimental measurements of the rubber boat in Fig. 4 indicate.

The metal boat in the image has characteristically low radiation temperatures. The lowest radiation temperature on the upper surfaces is $62 \mathrm{~K}$ in both polarizations, as this is reflecting the "cold" sky. The downward facing surfaces such as the hull appear with radiation temperatures around $\sim 177 \mathrm{~K}$ for the horizontal polarization and $250 \mathrm{~K}$ for the vertical polarization. The downward facing surfaces can also appear with low radiation temperatures, as "cold" sky radiation is reflected by the water on to the hull and then into the imager. The lower radiation temperatures of the metal surfaces in the horizontal polarization agree with measurements illustrated in Fig. 6.

The land in the maritime scenario has been modeled as a Lambertian scatterer with a reflectance of 5\% [31], as this most accurately describes the appearance of land covered in either grass or deciduous trees. The radiation temperature of the grass and trees is $274 \mathrm{~K}$, which is consistent with measurements by Wikner [55], [56]. The building in the simulation has vertical walls and a pitched roof and is modeled comprising entirely of brick and at a temperature of $288.15 \mathrm{~K}\left(15^{\circ} \mathrm{C}\right)$. The relatively low permittivity of brick gives a low reflectivity. Since any reflected radiation would originate from the horizon sky, which has a high radiation temperature, the radiation temperature 


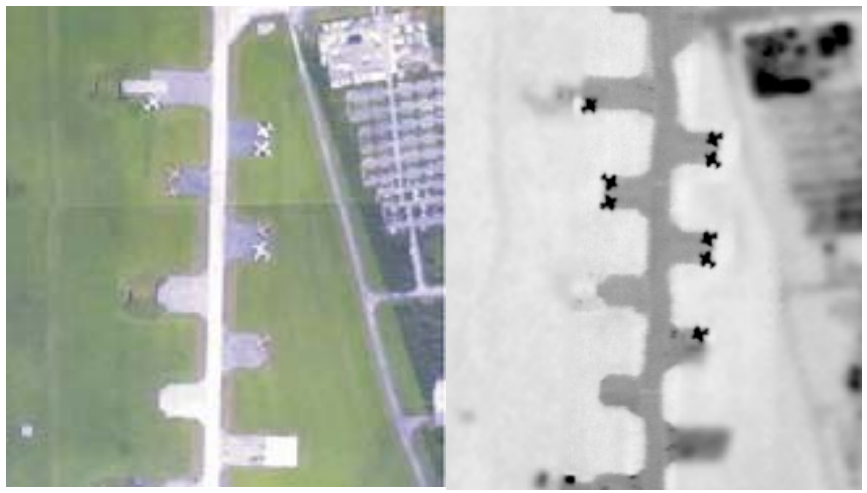

Fig. 7. Visible (left) and $90 \mathrm{GHz}$ (right) images of an airfield are shown from $100 \mathrm{~m}$ altitude [43]. The contrast in the millimeter-wave image here has been reversed; black denotes radiometrically 'cold.'

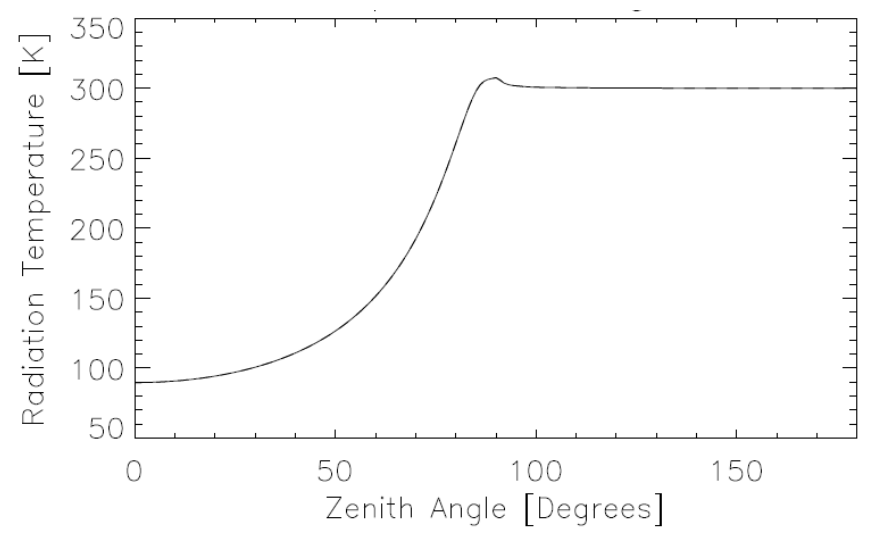

Fig. 8. Illumination radiation temperature profile for the sky (zenith angles: $0^{\circ}$ to $90^{\circ}$ ) and the ground (zenith angles: $90^{\circ}$ to $180^{\circ}$ ) for the airfield scenario, assuming grass has a Lambertian reflectance of $5 \%$.

of the vertical brick surface is also high, around $288.10 \mathrm{~K}$ indicated by the simulation.

\section{Viewing Vertically Downward From 100 m Altitude}

Probably the best combined visible and precisely calibrated experimental $90 \mathrm{GHz}$ images from [43] and [57] of an airfield from an altitude of $100 \mathrm{~m}$ are shown in Fig. 7. Here, the lowest radiation temperature in the scene is $98 \mathrm{~K}$, which is from a building with a large flat metal roof, having a size many times the diffraction limited imager resolution. To enhance the contrast definition of nonmetal objects in the scene, the contrast was adjusted to range from 250 to $298 \mathrm{~K}$.

To simulate the above experimental scenario, the illumination profile was created using the knowledge that measurements were made on a hot cloudless afternoon, when the temperature in the shade was in the region of $35^{\circ} \mathrm{C}$. Using the ITU recommended atmosphere [35] adapted for a ground temperature of $35{ }^{\circ} \mathrm{C}$, the illumination profile from the zenith to the horizon was modelled at $90 \mathrm{GHz}$, shown in Fig. 8. As the background is largely grass with a Lambertian reflectance of $5 \%$, this enables the remainder of the illumination profile from the horizon to the nadir to be created also shown in Fig. 8.

Simulations of the measurement scenario have been made assuming a PMMW imager located at an altitude of $100 \mathrm{~m}$,

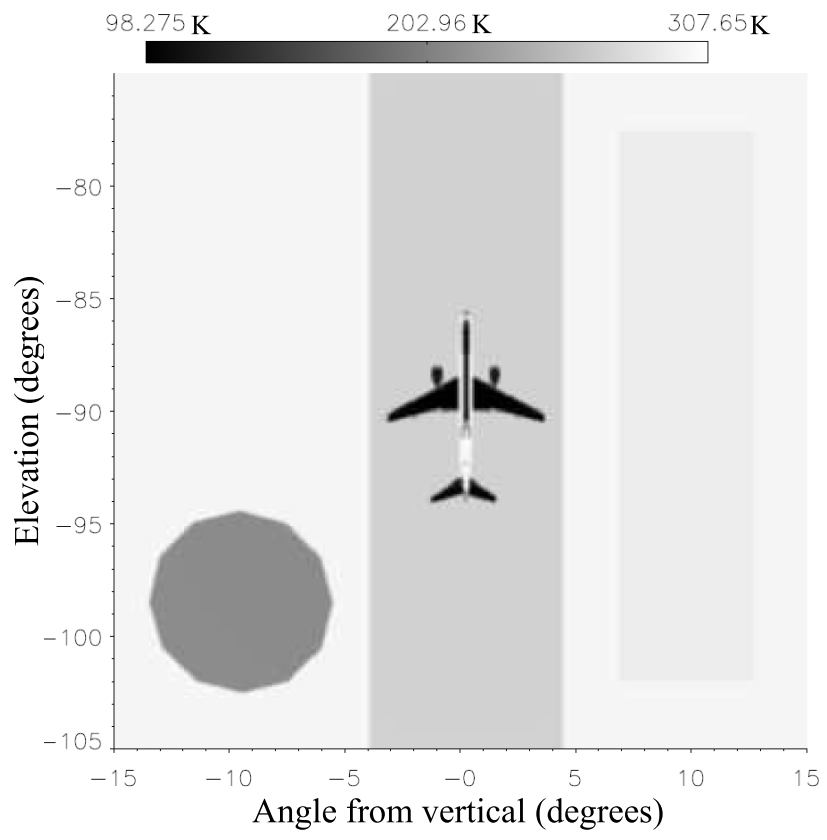

Fig. 9. Simulations of an airfield, showing a metal aircraft on a concrete runway, with a strip of asphalt to the right and a circular area of water to the left. Black denotes radiometrically 'cold.' The contrast bar above indicates the radiation temperatures.

having an aperture diameter of $1 \mathrm{~m}$, looking vertically down with a field of view of $30^{\circ} \times 30^{\circ}$. Materials in these simulations include metal for the aircraft, concrete for the runways, asphalt for roads with grass as the background material and a circular pool of water. The simulated image is shown in Fig. 9, in which the contrast has been reversed, to make black radiometrically "cold," to ease comparisons with the experimental data.

The grass in the background of the simulated image has a radiation temperature of $299.8 \mathrm{~K}$, which is compared with the measured radiation temperature of $298 \mathrm{~K}$. Grass has the highest radiation temperature in this scene, as it has the lowest reflectance.

A key feature of these images at the nadir is that aircraft, having large horizontal metal surface areas, appear with low radiation temperatures, as they reflect almost $100 \%$ of the "cold" zenith sky emission. This is shown in Fig. 9, where the radiation temperature of the aircraft is $98.3 \mathrm{~K}$, which can be compared with the experimental measurements of $98 \mathrm{~K}$ from Fig. 7. From the aperture diameter of $1 \mathrm{~m}$, the diffraction limited angle $\lambda / D$ is around $0.2^{\circ}$, so with an angular size of $1^{\circ}$ the metal wings have been resolved in the image.

Man-made material surfaces in the simulation have been modeled as specular reflectors and the concrete of the runway in the simulation has a radiation temperature of $269.6 \mathrm{~K}$, which can be compared with $\sim 271 \mathrm{~K}$ from the experimental measurements. Asphalt, which is shown as a long rectangle to the right in Fig. 9, has a radiation temperature of $291.5 \mathrm{~K}$, which can be compared to the experimental measurement value of $\sim 285 \mathrm{~K}$. A common feature of PMMW imaging from urban environments is that asphalt has a higher radiation temperature than concrete. 
For comparison a pool of water, assumed to have a physical temperature of $15{ }^{\circ} \mathrm{C}$, is shown in the simulation to have a radiation temperature of $212.3 \mathrm{~K}$. This has a radiation temperature intermediate between that of the grass and the metal of the aircraft, as the reflectance of water at normal incidence is calculated to be $\sim 38 \%$ at $15{ }^{\circ} \mathrm{C}$ from the relative permittivity and the Fresnel equations.

\section{Systematic Uncertainties in Radiation Temperatures}

For the experimental data, the uncertainties on the radiation temperatures are in the region of one half to a few kelvins, these arising from a combination of random noise and absolute calibration systematic uncertainties. For the comparison between experimental and simulated data, it is necessary to know the magnitudes of the systematic uncertainties. While it may not be possible to say with certainty the level of the systematic effects in the experiments, it is possible to estimate systematic effects in the simulation.

It was found that by varying the permittivities in Table I by up to $20 \%$ there was an almost linear inverse relationship to the object radiation temperature from the simulation. A $1 \%$ decrease in permittivity leads to an increase in the radiation temperature of up to $1 \mathrm{~K}$. So where the temperature estimated by the simulation differs from the measurement by say $5 \mathrm{~K}$, this could be due to an uncertainty in the permittivity of $5 \%$ or more. The levels of uncertainty in the measured permittivities are in the region of a few percent, so this can explain the difference between simulated and measured permittivities.

In the cases of permeable materials such as concrete, brick, some types of rock, and wood it may be difficult to know the precise value of the permittivity in an outdoor scenario. This is because weather conditions and the scenario will determine the level of water in these materials and increased water content raises the permittivity, as discussed in [34]. Measuring the permittivities of materials in a scene directly before or after image acquisition may help to bring down to the sub-kelvin level the agreement between simulation and experiment.

\section{Conclusion And Discussion}

A polarimetric ray tracing model for PMMW imaging has been presented, which models the atmosphere as an absorbing and emitting medium and models objects as specular or Lambertian reflectors. The material surfaces of the objects have been characterized using their measured permittivities and Lambertian reflectances. Comparisons in the $94 \mathrm{GHz}$ band between simulated and measured imagery have been made for three scenarios, a helicopter landing, a costal environment, and an airfield as viewed from directly above. The differences in the radiation temperatures between the simulated and measured imagery are within a few kelvins. This difference can be accounted for by the uncertainties in the material permittivities of objects in the scene. Improvements may be had by measuring precisely the dielectric properties of materials present in experimental imagery. Future work will concentrate on validation at other frequencies and for indoor security screening scenarios.
Future modeling activities might be conducted working directly with groups recording experimental data. Specific objects could then be investigated in more detail and uncertainties could be better quantified by presenting differential images, these being the differences between the experimental and the simulated imagery.

Modeling in this paper has been for imagers operating at $94 \mathrm{GHz}$ and having apertures of $\sim 1 \mathrm{~m}$. Since state-of-theart focal plane array-based imagers are as long as they are wide, this represents more or less the maximum tolerable imager size for most platforms. However, aperture synthesisbased imagers, potentially conformally deployable, would offer larger apertures, resulting in significantly improved image spatial resolutions [43], [44].

\section{REFERENCES}

[1] B. I. Hauss, H. H. Agravante, and S. Chaiken, "Advanced radiometric millimeter-wave scene simulation: ARMSS," Proc. SPIE, vol. 3064, pp. 182-194, Jun. 1997.

[2] M. R. Fetterman, J. Dougherty, and W. L. Kiser, "Scene simulation of millimeter-wave images," in Proc. IEEE Antennas Propag. Soc. Int. Symp., Jun. 2007, pp. 1493-1496.

[3] M. Murakowski, J. Wilson, J. Murakowski, G. Schneider, C. Schuetz, and D. Prather, "3D rendering of passive millimeter-wave scenes using modified open source software," Proc. SPIE, vol. 8022, pp. 80220B-1-80220B-8, May 2011.

[4] N. A. Salmon, R, Appleby, S. Price, and K. Mann, "Scene simulation of passive millimetre wave images of plastic and metal objects," Proc. SPIE, vol. 4491, pp. 52-56, Nov. 2001.

[5] N. A. Salmon, "W-band real-time passive millimeter-wave imager for helicopter collision avoidance," Proc. SPIE, vol. 3703, pp. 28-32, Apr. 1999.

[6] N. A. Salmon and R. Appleby, "Passive millimetre wave imaging through fog at $35 \mathrm{GHz}$ and $140 \mathrm{GHz}$," in Proc. 21st Int. Conf. Infr. Millim. Waves, Berlin, Germany, Jul. 1996.

[7] L. Mandel and E. Wolf, Optical Coherence and Quantum Optics, 1st ed. Cambridge, U.K.: Cambridge Univ. Press, 1995.

[8] M. Born and E. Wolf, Principles of Optics, 7th ed. Cambridge, U.K.: Cambridge Univ. Press, 2005.

[9] T. L. Wilson, K. Rohlfs, and S. Huettenmeister, Tools of Radio Astronomy, 6th ed. Berlin, Germany: Springer, 2013.

[10] N. A. Salmon, "Polarimetric passive millimeter-wave imaging scene simulation including multiple reflections of subjects and their backgrounds," Proc. SPIE, vol. 5989, pp. 598917-1-598917-5, Oct. 2005.

[11] N. A. Salmon, R. Appleby, and P. Coward, "Polarimetric passive millimetre wave imaging," Proc. SPIE, vol. 4373, pp. 82-85, Aug. 2001.

[12] N. A. Salmon and P. R. Coward, "Scattering in polarimetric millimetre-wave imaging scene simulation," Proc. SPIE, vol. 6211, pp. 621107-1-621107-8, May 2006.

[13] M. Peichl, "Fully-polarimetric passive MMW imaging systems for security applications," Proc. SPIE, vol. 7837, pp. 78370C-1-78370C-8, Oct. 2010.

[14] P. Lorrain, D. R. Corson, and F. Lorrain, Electromagnetic Fields and Waves, 3rd ed. San Francisco, CA, USA: Freeman, 1988.

[15] E. L. Stein et al., "Passive millimeter-wave cross polarization imaging and phenomenology," Proc. SPIE, vol. 7309, pp. 730902-1-730902-11, May 2009.

[16] C. Mann, "First demonstration of a vehicle mounted $250 \mathrm{GHz}$ real time passive imager," Proc. SPIE, vol. 7311, pp. 73110Q-1-73110Q-7, May 2009.

[17] R. Humphreys, P. Hirst, R. Heath, D. Elliner, N. Parker, and M. A. G. Smith, "Passive mm-wave imager using HTS Josephson junction detectors," Proc. SPIE, vol. 5619, pp. 59-69, Dec. 2004.

[18] Y. Zhang, Y. Jiang, J. Guo, Y. He, and H. Wang, "Application of millimeter-wave photonics technology in passive millimeter-wave imaging," Proc. SPIE, vol. 7854, pp. 785436-1-785436-6, Nov. 2010.

[19] R. Appleby, P. Coward, and J. N. Sanders-Reed, "Evaluation of a passive millimeter wave (PMMW) imager for wire detection in degraded visual conditions," Proc. SPIE, vol. 7309, pp. 73090A-1-73090A-8, Apr. 2009. 
[20] A. Stogryn, "Equations for calculating the dielectric constant of saline water," IEEE Trans. Microw. Theory Techn., vol. MTT-19, no. 8, pp. 733-736, Aug. 1971.

[21] L. A. Klein and C. T. Swift, "An improved model for the dielectric constant of sea water at microwave frequencies," IEEE Trans. Antennas Propag., vol. AP-25, no. 1, pp. 104-111, Jan. 1977.

[22] H. J. Liebe, G. A. Hufford, and T. Manabe, "A model for the complex permittivity of water at frequencies below $1 \mathrm{THz}$, Int. J. Infr. Millim. Waves, vol. 12, no. 7, pp. 659-674, 1991.

[23] W. Miller, "Dielectric properties of natural media," in Thermal Microwave Radiation: Applications for Remote Sensing (IET Electromagnetic Waves Series), vol. 52, C. Mätzler, Ed. Edison, NJ, USA: IET, 2006, ch. 5.

[24] K. Sato and T. Manabe, "Measurements of reflection characteristics and refractive indices of interior construction materials in millimeter-wave bands," in Proc. IEEE 45th Veh. Technol. Conf., Jul. 1995, pp. 449-453.

[25] R. Piesiewicz, C. Jansen, S. Wietzke, D. Mittleman, M. Koch, and T. Kürner, "Properties of building and plastic materials in the $\mathrm{THz}$ range," Int. J. Infr. Millim. Waves, vol. 28, no. 5, pp. 363-371, 2007.

[26] B. Nashashibi, F. T. Ulaby, and K. Sarabandi, "Measurement and modeling of the millimeter-wave backscatter response of soil surfaces," IEEE Trans. Geosci. Remote Sens., vol. 34, no. 2, pp. 561-572, Mar. 1996.

[27] F. T. Ulaby, "Microwave dielectric properties of dry rocks," IEEE Trans. Geosci. Remote Sens., vol. 28, no. 3, pp. 325-336, May 1990.

[28] K. Sarabandi, E. S. Li, and A. Nashashibi, "Modeling and measurements of scattering from road surfaces at millimeter-wave frequencies," IEEE Trans. Antennas Propag., vol. 45, no. 11, pp. 1679-1688, Nov. 1997.

[29] A. R. von Hippel, Dielectric Materials and Applications. Norwood, MA, USA: Artech House, 1995.

[30] A. J. Gatesman et al., "Terahertz behavior of optical components and common materials," Proc. SPIE, vol. 6212, pp. 62120E-1-62120E-12, May 2006.

[31] A. W. England and J. F. Galantowicz, "A volume emission, model for the radiobrightness of prairie grass," in Proc. Geosci. Remote Sens. Symp. (IGARSS), Surf. Atmos. Remote Sens., Technol., Data Anal. Interpretation, vol. 4. Aug. 1994, pp. 2463-2465.

[32] T. J. Hewson, "Airborne measurements of forest and agricultural land surface emissivity at millimeter wavelengths," IEEE Trans. Geosci. Remote Sens., vol. 39, no. 2, pp. 393-400, Feb. 2001.

[33] N. A. Salmon, "Polarimetric scene simulation in millimeter-wave radiometric imaging," Proc. SPIE, vol. 5410, pp. 260-270, Aug. 2004.

[34] F. T. Ulaby and D. G. Long, Microwave Radar and Radiometric Remote Sensing. Norwood, MA, USA: Artech House, 2015.

[35] Reference Standard Atmospheres, document ITU-R P.835-3, International Telecommunications Union Recommendation, 1999.

[36] Attenuation by Atmospheric Gases, document ITU-R P.676-8, International Telecommunications Union Recommendation, 2009.

[37] Attenuation Due to Clouds and Fog, document ITU-R P.840-3, International Telecommunications Union Recommendation, 1999.

[38] Specific Attenuation Model for Rain for Use in Prediction Methods, document ITU-R P.838-2, International Telecommunications Union Recommendation, 2003.

[39] X. Y. Dong, H. Y. Chen, and D. H. Guo, "Microwave and millimeterwave attenuation in sand and dust storms," IEEE Antennas Wireless Propag. Lett., vol. 10, pp. 469-471, 2011.

[40] M.-M. Chiou and J.-F. Kiang, "Attenuation of millimeter-wave in a sand and dust storm," IEEE Geosci. Remote Sens. Lett., vol. 13, no. 8, pp. 1094-1098, Aug. 2016.

[41] S. T. Fiorino et al., "Lab measurements to support modeling terahertz propagation in brownout conditions," Proc. SPIE, vol. 7671, pp. 76710W-1-76710W-11, Apr. 2010.

[42] D. Wikner, "Millimeter-wave propagation measurement through a dust tunnel," U.S. Army Res. Lab., Adelphi, MD, USA, Tech. Rep. ARLTR-4399, Mar. 2008.

[43] M. Peichl, H. Süß, and S. Dill, "High-resolution passive millimeter-wave imaging technologies for reconnaissance and surveillance," Proc. SPIE, vol. 5077, pp. 77-86, Aug. 2003.

[44] N. A. Salmon, "Passive millimeter wave imagers for safety and security," Proc. SPIE, pp. 1-4, Aug. 2008, doi: 10.1117/2.1200808.1236.

[45] N. A. Salmon, "3-D radiometric aperture synthesis imaging," IEEE Trans. Microw. Theory Techn., vol. 63, no. 11, pp. 3579-3587, Nov. 2015.

[46] R. Humphreys et al., "Performance of $94 \mathrm{GHz}$ receivers for passive imaging," Proc. SPIE, vol. 6548, pp. 65480H-1-65480H-10, Apr. 2007.

[47] D. M. Pozar, Microwave Engineering, 4th ed. Hoboken, NJ, USA: Wiley, 2012.
[48] F. Colucci, "Digging out from brownout," Safety Spotlight, Vertiflite, vol. 53, no. 1, pp. 51-55, 2007.

[49] C. S. Ruf, C. T. Swift, A. B. Tanner, and D. M. Le Vine, "Interferometric synthetic aperture microwave radiometry for the remote sensing of the Earth," IEEE Trans. Geosci. Remote Sens., vol. GRS-26, no. 5, pp. 597-611, Sep. 1988.

[50] D. A. Wikner, "Passive millimeter-wave imagery of helicopter obstacles in a sand environment," Proc. SPIE, vol. 6211, pp. 621103-1-621103-8, May 2006.

[51] Y. G. M. Hurtaud, "Millimetre wave propagation over the sea," NATO Technol. Group Rep., Centre d'Electronique de l'Armement (CELAR), Rennes, France, Tech. Rep. AC/243 (Panel 3) TR/3, Oct. 1990.

[52] P. Yao, C. A. Schuetz, and S. Shi, "Development of high speed modulator for w-band millimeter-wave imaging system," Proc. SPIE, vol. 7309, pp. 7309L-1-7309L-11, May 2009.

[53] R. Martin et al., "Design and performance of a distributed aperture millimeter-wave imaging system using optical upconversion," Proc. SPIE, vol. 7309, pp. 730908-1-730908-10, May 2009.

[54] P. Coward and R. Appleby, "Comparison of passive millimetre-wave and IR imagery in a nautical environment," Proc. SPIE, vol. 7309, pp. 730904-1-730904-8, May 2009.

[55] D. A. Wikner, "Polarimetric radiometry of natural scenes," Proc. SPIE, vol. 4719, pp. 391-397, Jul. 2002.

[56] D. A. Wikner, "Millimeter-wave radiometric measurements of a treeline and building for aircraft obstacle avoidance," Proc. SPIE, vol. 5077, pp. 7-15, 2003.

[57] M. Peichl, S. Dill, and H. Süß, "Microwave radiometry-Imaging technologies and applications," in Proc. WFMN, Chemnitz, Germany, 2007, pp. 75-83. [Online]. Available: http://archiv.tuchemnitz.de/pub/2007/0210/

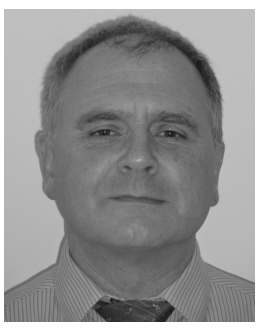

Neil A. Salmon began innovating millimeter wave systems at Imperial College London, London, U.K. in 1984 , to investigate tokamak plasma instabilities for nuclear fusion research at the Joint European Torus (JET). Developing novel heterodyne radiometer diagnostics to measure plasma electron cyclotron emission, he identified temperature changes localized to the rational flux surfaces and at the last, closed flux surface in the plasma edge regions played determining roles in global plasma stability. Following this, he laid the foundation to develop the heterodyne radiometer into the central diagnostic for the study of fast and localized temperature phenomena in JET. In 1990, he moved to the Max Planck Institute for Plasma Physics, Garching, Germany, to develop a suite of millimeter wave spectrometers (heterodyne radiometers, a Michelson interferometer, and a grating polychromator). He used these to make the first measurements of the electron temperature on the ASDEX-Upgrade tokamak; the diagnostics continue to this day to deliver new information about tokamak phenomena.

In 1994, he moved to the Defence Research Agency (now QinetiQ), Malvern, U.K., where he worked in a team to demonstrate the capabilities of millimeter waves for communications and all-weather imaging and screening for concealed threats on persons. This involved developing and trialing focal plane array passive imagers (from a few to several hundred $\mathrm{GHz}$ ) together with scene simulations and parametric models to analyze and quantify performance. The work culminated in developing the world's first mechanical scanning passive millimeter wave imager, deployment of follow-ons from this for use on helicopters and the development of security screening portals to screen people and vehicles.

In 2009, he set up a collaboration with the University of Manchester Manchester, U.K., in which he developed proof-of-concept aperture synthesis imaging systems at 22, 94, and $183 \mathrm{GHz}$, demonstrating real-time imaging capabilities using a technology to enable conformal sensor deployment on air platforms and confined spaces of entrances for security screening. $\mathrm{He}$ then went on to develop the M.Sc. course in radio imaging and sensing, teaching about radar technology and techniques. In 2012, he moved to Manchester Metropolitan University, Manchester, U.K., where he demonstrated how aperture synthesis could generate 3-D images. He continues to pursue: algorithms for passive 3-D imaging, techniques for active stand-off non-imaging polarimetry, and millimeter waves for medical applications. During his time at this institute, he has developed the applied physics B.Sc. lecture and tutorial courses. 